\title{
Análise comparativa da competividade do Brasil e EUA no mercado internacional da carne bovina
}

\author{
Matheus Dhein Dill, Vitor Francisco Dalla Corte ${ }^{2}$, Júlio Otávio Jardim Barcellos ${ }^{3}$, \\ Maria Eugênia Andrighetto Canozzi ${ }^{4}$, Tamara Esteves de Oliveira ${ }^{5}$
}

\section{RESUMO}

O Brasil e os EUA estão entre os principais produtores mundiais de carne bovina. Entretanto, distorções no mercado alimentar decorrentes da presença de barreiras comerciais podem comprometer a competitividade desses países. O objetivo deste trabalho foi verificar a competitividade da carne bovina brasileira e norte-americana, no mercado internacional, entre 1990 e 2008. Para isso, foi utilizado o Índice de Competitividade Revelada (CR) para inferir sobre os efeitos que subsídios, acordos comercias e barreiras sanitárias exercem sobre a competitividade da carne bovina dos respectivos países. Os resultados indicaram que o Brasil obteve vantagens competitivas no período de 1991 a 2008, enquanto que os EUA apresentaram vantagens entre 1993 e 2003. Os acordos comerciais elevaram a competitividade dos países envolvidos, contudo ocorreram diminuições dos índices quando problemas sanitários foram identificados. Em suma, os EUA, mesmo com os altos subsídios fornecidos aos produtores rurais, apresentou desempenho inferior em comparação ao Brasil no mercado mundial da carne bovina.

Palavras-chave: livre comércio, vantagem comparativa, política pública.

\section{ABSTRACT \\ Comparative analysis of the competitiveness of Brazil and the USA in the international beef market}

Brazil and the USA are among the leading producers of beef. However, trade barriers can bring about distortions in the food market, compromising competitiveness. The objective of this study was to evaluate the competitiveness of beef from Brazil and the USA in the international market from 1990 to 2008. The Revealed Competitiveness Index was used to discuss the effects of subsidies, trade agreements and sanitary barriers on the competitiveness of beef in the respective countries. The results indicate that Brazil had competitive advantages in the period 1991 to 2008, while The USA had advantages in the period 1993 to 2003. Trade agreements increased the competitiveness of these countries, however there was decrease in rates when sanitary problems were identified. Therefore, even with high subsidies provided to farmers, the USA presented lower performance compared to Brazil in the beef world market.

Key words: free trade, comparative advantage, public policy.

\footnotetext{
Recebido para publicação em 14/12/2012 e aprovado em 14/06/2013.

${ }^{1}$ Médico Veterinário, Mestre. Centro de Estudos e Pesquisas em Agronegócios (CEPAN), Programa de Pós-Graduação em Agronegócios, Universidade Federal do Rio Grande do Sul (UFRGS), Avenida Bento Gonçalves, 7712, Bairro Agronomia, 91540-000, Porto Alegre, Rio Grande do Sul, Brasil. matheusdill@ hotmail.com (autor para correspondência); ${ }^{2}$ Economista, Mestre. Centro de Estudos e Pesquisas em Agronegócios (CEPAN), Programa de Pós-Graduação em Agronegócios, Universidade Federal do Rio Grande do Sul (UFRGS), Avenida Bento Gonçalves, 7712, Bairro Agronomia, 91540-000, Porto Alegre, Rio Grande do Sul, Brasil. vitordallacorte@gmail.com

${ }_{3}^{3}$ Médico Veterinário, Doutor. Departamento de Zootecnia, Programa de Pós-Graduação em Zootecnia, Programa de Pós-Graduação em Agronegócios, Universidade Federal do Rio Grande do Sul (UFRGS), Avenida Bento Gonçalves, 7712, Bairro Agronomia, 91540-000, Porto Alegre, Rio Grande do Sul, Brasil. julio.barcellos@ufrgs.br

${ }^{4}$ Médica Veterinária, Mestre. Departamento de Zootecnia, Programa de Pós-Graduação em Zootecnia, Universidade Federal do Rio Grande do Sul (UFRGS), Avenida Bento Gonçalves, 7712, Bairro Agronomia, 91540-000, Porto Alegre, Rio Grande do Sul, Brasil. mecanozzi@yahoo.com.br

${ }_{5}^{5}$ Médica Veterinária, Mestre. Centro de Estudos e Pesquisas em Agronegócios (CEPAN), Universidade Federal do Rio Grande do Sul (UFRGS), Avenida Bento Gonçalves, 7712, Bairro Agronomia, 91540-000, Porto Alegre, Rio Grande do Sul, Brasil. tamaraesteves@yahoo.com.br
} 


\section{INTRODUÇÃO}

Problemas relacionados com as barreiras comerciais, como os subsídios fornecidos aos produtores rurais nos países desenvolvidos, além das alegações de ordem sanitária no mercado internacional da carne bovina, têm gerado discussões nos debates da Organização Mundial do Comércio.

O crescente fluxo do comércio internacional, advindo da liberalização comercial, tem fortalecido gradativamente a economia dos países em desenvolvimento, proporcionando ganhos à população (Balassa, 1965; Milner \& Kubota, 2004). Ademais, o país que busca ser competitivo deve se especializar na produção de bens que possuem fatores de produção em abundância e importar produtos escassos no país. Diante desse pressuposto, o livre comércio tenderá a beneficiar os países envolvidos nas trocas comerciais. Contudo, os tributos (impostos) e prêmios (subsídios) podem diminuir os efeitos das vantagens comparativas que o país possui (Ricardo, 1817).

Desse modo, as políticas protecionistas dos países desenvolvidos ainda representam um entrave ao livre comércio (Opp, 2010). Os EUA, maior produtor mundial de carne bovina (USDA, 2012), fornece grandes quantias de subsídios aos produtores rurais, consequência dos altos custos de produção, tendo como propósito a proteção de seus mercados (Bismarck, 2004; MAPA, 2007). Por outro lado, o Brasil, segundo maior produtor de carne bovina (USDA, 2012) é reconhecido pelo pequeno apoio governamental fornecido aos seus produtores rurais (OECD, 2005). Apesar disso, é um forte competidor na produção de carnes, devido aos avanços em termos de produtividade, expansão das fronteiras agropecuárias e baixo custo de produção (MAPA, 2007; Martinelli et al., 2010), além de ter a maior biocapacidade mundial para produzir alimentos (WWF, 2010). Mesmo assim, tem enfrentado barreiras comerciais para exportar seus produtos (OECD, 2005).

Tendo em vista o atual cenário, este trabalho utilizou o Índice de Competitividade Revelada (CR) para verificar, separadamente, a competitividade da carne bovina do Brasil e dos EUA, no período de 1990 a 2008. Dessa forma, procurou-se inferir sobre os efeitos que as barreiras comerciais (subsídios e questões sanitárias), assim como os acordos de cooperação entre países e blocos econômicos, influenciaram na competitividade desses países no comércio internacional da carne bovina. Nesse contexto, questionou-se: Qual país é mais competitivo no mercado internacional da carne bovina: Brasil ou Estados Unidos da América?

\section{MATERIAL E MÉTODOS}

A teoria das Vantagens Comparativas, como determinante do comércio internacional, foi desenvolvida por
David Ricardo em 1817. Seus estudos retrataram as influências que os fatores de produção (terra, capital e trabalho) exercem sobre o desempenho das nações, sendo que o país que possuir recursos superiores apresentará vantagem em comparação ao outro (Ricardo, 1817). Balassa (1965) criou o Índice de Vantagem Comparativa Revelada (IVCR) com embasamento na teoria de Ricardo (1817). O Índice é calculado através de dados comerciais de países ou blocos econômicos, utilizando informações das exportações do país e do mundo de determinado produto.

Utilizando a mesma base conceitual do IVCR, Carvalho (2001) propôs o Índice de Competitividade Revelada (CR), que engloba todo o comércio e não somente as exportações. Pelo fato do Brasil e Estados Unidos serem importadores e exportadores de carne bovina, este trabalho utilizou o CR para analisar, separadamente, a competitividade de ambos os países no mercado internacional desse produto, entre 1990 e 2008. Os resultados foram obtidos através da seguinte expressão:

$$
C R_{k i}=\ln \left[\frac{X_{k i} / X_{k r}}{X_{m i} / X_{m r}} / \frac{M_{k i} / M_{k r}}{M_{m i} / M_{m r}}\right]
$$

em que:

$k=$ carne bovina;

$C R k i$ = Índice de Competitividade Revelada da carne bovina do país "i";

$X k i=$ valor total das exportações do produto " $\mathrm{k}$ ", do país "i";

$X k r=$ valor total das exportações mundiais do produto "k", menos as do país "i";

$X m i=$ valor total das exportações do país "i”" (agregado), menos as suas exportações do produto "k";

$X m r=$ valor total das exportações mundiais (agregado), menos as do país "i" e do produto "k";

$M k i=$ valor total das importações do produto "k", do país “ "i”; $M k r=$ valor total das importações mundiais do produto "k", menos as do país "i";

$M m i=$ valor total das importações do país "i” (agregado), menos as suas importações do produto " $k$ ";

$M m r=$ valor total das importações mundiais (agregado), menos as do país "i" e do produto "k".

Conforme Carvalho (2001), se o CRki > 0, o país possui Vantagem Competitiva Revelada no comércio de " $k$ " e, caso apresente $C R k i<0$, desvantagem. Dessa forma, o CR possibilita verificar se um país tem vantagem competitiva para determinado produto, confrontando a sua participação nas exportações e importações a nível nacional e mundial em relação às exportações e importações de um país para o mesmo produto (Machado et al., 2007). 
Os valores nominais (US\$) das exportações e importações para calcular o índice CR da carne bovina são oriundos da Food and Agriculture Organization of the United Nations (FAO, 2011), enquanto que os valores das importações e exportações de todos os produtos são provenientes da Organização Mundial do Comércio (WTO, 2011) (Tabelas 1, 2 e 3).

\section{RESULTADOS E DISCUSSÃO}

Analisando os índices de Competitividade Revelada (CR), os resultados demonstraram que o Brasil apresentou resultados superiores em comparação com os EUA. O ano de 1990 foi o único em que o Brasil mostrou desvantagem no mercado internacional da carne bovina $(C R=-0,185)$, mas, mesmo assim, uma posição superior em comparação aos EUA (CR = -0,325) (Figura 1).

O desempenho negativo do Brasil pode ser consequência do déficit comercial de US\$ -39,69 milhões em carne bovina (FAO, 2011). Outro fator que pode ter contribuído foi os resultados negativos remanescentes da década de 1980, período em que o país enfrentou problemas com a dívida externa e com o processo inflacionário (Carvalho, 2001).

Foi a partir de 1991 que o Brasil passou a apresentar resultados positivos no CR. O país exportou cerca de US\$ 430 milhões em carne bovina, um aumento de 57,34\% em relação ao ano anterior, tendo como principais compradores a Angola, EUA, Reino Unido, Itália e Espanha (FAO, 2011). Outro fator que pode ter contribuído para o aumento do índice foi a formação do Mercado Comum do Sul (MERCOSUL), onde as vendas de todos os produtos do Brasil para os países membros tiveram um crescimento de 117\% até o ano de 1998 (Carvalho, 1999).

Os EUA obtiveram resultados negativos nos anos de 1990, 1991 e 1992 (CR = -0,325; -0,357; -0,363, respectivamente), mesmo com os altos subsídios governamentais fornecidos para os produtores rurais, que giraram em torno de $16 \%$ ao ano em relação à renda bruta da agricultura (OECD, 2010). Em 1993, os EUA iniciaram um processo de crescimento das exportações e diminuição das importa-

Tabela 1. Valores nominais (US\$) das exportações e importações de carne bovina e todos os produtos no mundo, de acordo com os dados da FAO (2011) e WTO (2011)

\begin{tabular}{|c|c|c|c|c|}
\hline \multirow[b]{2}{*}{ Ano } & \multicolumn{4}{|c|}{ Mundo (US\$ em mil) } \\
\hline & $\begin{array}{l}\text { Exportação de } \\
\text { carne bovina }\end{array}$ & $\begin{array}{c}\text { Exportação de } \\
\text { todos os produtos }\end{array}$ & $\begin{array}{l}\text { Importação de } \\
\text { carne bovina }\end{array}$ & $\begin{array}{l}\text { Importação de } \\
\text { todos os produtos }\end{array}$ \\
\hline 1981 & 9.482 .255 & 2.010 .000 .000 & 9.177 .826 & 2.066 .000 .000 \\
\hline 1982 & 8.808 .452 & 1.883 .000 .000 & 9.252 .675 & 1.941 .000 .000 \\
\hline 1983 & 8.569 .604 & 1.846 .000 .000 & 9.282 .700 & 1.890 .000 .000 \\
\hline 1984 & 7.713 .045 & 1.956 .000 .000 & 8.131 .327 & 2.014.000.000 \\
\hline 1985 & 7.531 .586 & 1.954 .000 .000 & 8.244 .721 & 2.015 .000 .000 \\
\hline 1986 & 9.127 .932 & 2.138 .000 .000 & 9.892 .955 & 2.206 .000 .000 \\
\hline 1987 & 11.003 .918 & 2.516 .000 .000 & 11.499 .145 & 2.582 .000 .000 \\
\hline 1988 & 12.440 .775 & 2.869 .000 .000 & 12.790 .066 & 2.964 .000 .000 \\
\hline 1989 & 13.367 .984 & 3.098 .000 .000 & 14.110 .265 & 3.201 .000 .000 \\
\hline 1990 & 14.584 .033 & 3.449.000.000 & 15.974 .487 & 3.550 .000 .000 \\
\hline 1991 & 15.698 .737 & 3.515 .000 .000 & 16.517 .750 & 3.632 .000 .000 \\
\hline 1992 & 16.919 .204 & 3.766 .000 .000 & 17.587 .447 & 3.881 .000 .000 \\
\hline 1993 & 15.608 .986 & 3.782 .000 .000 & 16.161 .488 & 3.875 .000 .000 \\
\hline 1994 & 16.774 .279 & 4.326 .000 .000 & 17.053 .424 & 4.428 .000 .000 \\
\hline 1995 & 17.371 .820 & 5.164 .000 .000 & 17.335 .535 & 5.283 .000 .000 \\
\hline 1996 & 14.536 .806 & 5.403 .000 .000 & 15.002 .155 & 5.544 .000 .000 \\
\hline 1997 & 14.881 .540 & 5.591 .000 .000 & 15.183 .014 & 5.737 .000 .000 \\
\hline 1998 & 14.489 .272 & 5.501 .000 .000 & 14.971 .798 & 5.681 .000 .000 \\
\hline 1999 & 15.558 .808 & 5.712 .000 .000 & 15.431 .010 & 5.921 .000 .000 \\
\hline 2000 & 15.412 .070 & 6.456 .000 .000 & 15.399 .775 & 6.724 .000 .000 \\
\hline 2001 & 13.648 .487 & 6.483 .000 .000 & 13.700 .884 & 6.191 .000 .000 \\
\hline 2002 & 15.127 .398 & 6.742 .000 .000 & 14.965 .042 & 6.492 .000 .000 \\
\hline 2003 & 17.973 .255 & 7.867.000.000 & 17.559 .945 & 7.586.000.000 \\
\hline 2004 & 20.296 .789 & 9.568 .000 .000 & 19.718 .233 & 9.218 .000 .000 \\
\hline 2005 & 23.268 .500 & 10.855 .000 .000 & 21.795 .989 & 1.0489.000.000 \\
\hline 2006 & 26.164.909 & 12.113 .000 .000 & 24.805 .215 & 12.437 .000 .000 \\
\hline 2007 & 28.967 .735 & 14.000 .000 .000 & 28.320 .397 & 14.300.000.000 \\
\hline 2008 & 35.318 .947 & 16.116 .000 .000 & 32.664 .063 & 16.520 .000 .000 \\
\hline
\end{tabular}


ções de carne bovina, o que colaborou para o início de um período de resultados positivos revelados pelo CR.

Entre os anos de 1994 e 1997, o Brasil diminuiu o CR da carne bovina. Esta perda de competitividade foi influenciada pela diminuição das exportações de carne bovina, devido à sobrevalorização da moeda brasileira nos primeiros anos do Plano Real (MAPA, 2007) e ao aumento do consumo interno decorrente da estabilidade da economia interna (Paula \& Faveret Filho, 2001). Neste período, o Brasil também enfrentou um pico no volume de subsídios destinados aos produtores dos EUA, garantidos pela reversão da Lei Agrícola de 1996 (Jank et al., 2004).

O período de vantagem dos EUA no mercado internacional da carne bovina iniciou-se em 1993 e manteve-se até 2003. No período de 1995 a 2003, os subsídios diretos fornecidos aos pecuaristas somaram cerca de US\$ 2,6 bilhões, sendo o ano de 2002 o de maior participação governamental na recente história dos EUA, com o fornecimento de cerca de US\$ 976 milhões (EWG, 2011).
Em 1994, foi constituído o Tratado Norte-Americano de Livre Comércio (NAFTA), visando reduzir as barreiras comerciais entre os países membros. A partir desse acordo, Canadá e México se tornaram mercados importantes para a carne bovina norte-americana (Henneberry \& Mutondo, 2009). Isso permitiu aos EUA ser, até 2003, o principal exportador mundial de carne bovina, contudo, surtos de encefalopatia espongiforme bovina (BSE) deterioraram a participação desse país no comércio mundial (USDA, 2010). O Canadá e o México, a partir dos surtos de BSE, proibiram a importação de carne bovina dos Estados Unidos, o que diminuiu a competitividade do país (Henneberry \& Mutondo, 2009).

Entre 2004 e 2008, o índice revelou que os EUA obtiveram desvantagem $(\mathrm{CR}=-0,567 ;-0,409 ;-0,258 ;-0,168$; 0,165, para 2004, 2005, 2006, 2007 e 2008, respectivamente) no mercado internacional da carne bovina, que pode estar relacionado com a diminuição dos subsídios fornecidos aos produtores (EWG, 2011).

Tabela 2. Valores nominais (US\$) das exportações e importações de carne bovina e todos os produtos no Brasil, de acordo com os dados da FAO (2011) e WTO (2011)

\begin{tabular}{|c|c|c|c|c|}
\hline \multirow[b]{2}{*}{ Ano } & \multicolumn{4}{|c|}{ Brasil (US\$ em mil) } \\
\hline & $\begin{array}{l}\text { Exportação de } \\
\text { carne bovina }\end{array}$ & $\begin{array}{c}\text { Exportação de } \\
\text { todos os produtos }\end{array}$ & $\begin{array}{l}\text { Importação de } \\
\text { carne bovina }\end{array}$ & $\begin{array}{c}\text { Importação de } \\
\text { todos os produtos }\end{array}$ \\
\hline 1980 & 267.425 & 20.132 .000 & 89.854 & 24.961 .000 \\
\hline 1981 & 442.866 & 23.293 .000 & 72.719 & 24.079 .000 \\
\hline 1982 & 462.821 & 20.175 .000 & 19.558 & 21.069 .000 \\
\hline 1983 & 540.683 & 21.899 .000 & 23.084 & 16.801 .000 \\
\hline 1984 & 544.535 & 27.005 .000 & 29.022 & 15.210 .000 \\
\hline 1985 & 544.834 & 25.639 .000 & 39.040 & 14.332 .000 \\
\hline 1986 & 404.917 & 22.349 .000 & 396.073 & 15.557 .000 \\
\hline 1987 & 461.304 & 26.224 .000 & 141.317 & 16.581 .000 \\
\hline 1988 & 660.856 & 33.494 .000 & 19.230 & 16.055 .000 \\
\hline 1989 & 342.527 & 34.383 .000 & 249.123 & 19.875 .000 \\
\hline 1990 & 246.917 & 31.414 .000 & 286.608 & 22.524 .000 \\
\hline 1991 & 430.547 & 31.620 .000 & 116.401 & 22.950 .000 \\
\hline 1992 & 638.624 & 35.793 .000 & 121.447 & 23.068 .000 \\
\hline 1993 & 601.967 & 38.555 .000 & 36.090 & 27.740 .000 \\
\hline 1994 & 577.729 & 43.545 .000 & 116.645 & 35.997 .000 \\
\hline 1995 & 492.800 & 46.506 .000 & 179.790 & 54.137 .000 \\
\hline 1996 & 439.225 & 47.747 .000 & 180.717 & 56.792 .000 \\
\hline 1997 & 436.584 & 52.994 .000 & 207.351 & 63.291 .000 \\
\hline 1998 & 593.070 & 51.140 .000 & 160.568 & 61.135 .000 \\
\hline 1999 & 808.458 & 48.011 .000 & 72.465 & 51.909 .000 \\
\hline 2000 & 783.188 & 55.086 .000 & 100.198 & 59.053 .000 \\
\hline 2001 & 1.008 .676 & 58.223 .000 & 58.275 & 58.640 .000 \\
\hline 2002 & 1.089 .924 & 60.362 .000 & 68.389 & 49.716 .000 \\
\hline 2003 & 1.507 .643 & 73.084 .000 & 60.177 & 50.859 .000 \\
\hline 2004 & 2.428 .669 & 96.678 .000 & 73.332 & 66.433 .000 \\
\hline 2005 & 2.964 .685 & 118.529 .000 & 80.380 & 77.628 .000 \\
\hline 2006 & 3.816 .876 & 137.808 .000 & 66.618 & 95.838 .000 \\
\hline 2007 & 4.263 .834 & 160.649 .000 & 95.042 & 126.645 .000 \\
\hline 2008 & 4.991 .491 & 197.942 .000 & 123.593 & 182.377 .000 \\
\hline
\end{tabular}

Rev. Ceres, Viçosa, v. 60, n.6, p. 765-771, nov/dez, 2013 
As exportações brasileiras, a partir de 1998, iniciaram uma nova fase de crescimento que se estendeu até 2008. As negociações entre MERCOSUL e União Europeia, com a diminuição das barreiras comerciais (Waquil et al., 2004) e com a desvalorização da moeda brasileira, tornaram os produtos brasileiros mais competitivos no mercado internacional (Reis, 2008), contribuindo para o aumento da competitividade revelada $(C R=1,09)$ em 1999.

Em 2000, o Índice brasileiro revelou uma ligeira queda $(\mathrm{CR}=0,93)$, consequência dos surtos de febre aftosa, em que milhares de animais foram sacrificados para controlar a disseminação da doença. Em maio de 2000, Rio Grande do Sul e Santa Catarina haviam suspendido a vacinação para conquistar o Certificado de Zona Livre de Febre Aftosa Sem Vacinação, junto à OIE. Entretanto, o reaparecimento da doença neste mesmo ano resultou na suspensão do certificado que garantiria aos Estados o status de Zona Livre Sem Vacinação (MAPA, 2001), resultando na paralisação dos contratos de exportação de car- ne e derivados para o Chile, Arábia Saudita, Rússia e países da União Europeia (Andreatta, 2003). Outro fator que influenciou a diminuição do CR foi o déficit da balança comercial brasileira (WTO, 2011).

A partir de 2002, as exportações brasileiras de carne bovina tiveram um crescimento expressivo, favorecido pelo cenário mundial. Conforme Polaquini et al. (2006), esse aumento foi resultado i) da diminuição das áreas com febre aftosa no país, ii) da implantação de sistemas de controle de qualidade para atender os mercados mais exigentes, iii) das deficiências no abastecimento de carne bovina decorrentes de surtos BSE na UE e iv) das crises econômicas e sanitárias na Argentina. Nesse período, a Argentina, um dos principais produtores mundiais de carne bovina, perdeu espaço no mercado internacional devido aos surtos de febre aftosa e sobrevalorização da moeda, proporcionando ao Brasil um crescimento nas exportações de carne bovina para os EUA (Paula \& Faveret Filho, 2001). Esse cenário permitiu ao Brasil aumentar,

Tabela 3. Valores nominais (US\$) das exportações e importações de carne bovina e todos os produtos nos EUA, de acordo com os dados da FAO (2011) e WTO (2011)

\begin{tabular}{|c|c|c|c|c|}
\hline \multirow[b]{2}{*}{ Ano } & \multicolumn{4}{|c|}{ EUA (US\$ em mil) } \\
\hline & $\begin{array}{c}\text { Exportação de } \\
\text { carne bovina }\end{array}$ & $\begin{array}{c}\text { Exportação de } \\
\text { todos os produtos }\end{array}$ & $\begin{array}{l}\text { Importação de } \\
\text { carne bovina }\end{array}$ & $\begin{array}{c}\text { Importação de } \\
\text { todos os produtos }\end{array}$ \\
\hline 1980 & 258.136 & 225.566 .000 & 1.786 .105 & 256.984 .000 \\
\hline 1981 & 309.521 & 238.715 .000 & 1.418 .447 & 273.352 .000 \\
\hline 1982 & 386.701 & 216.442 .000 & 1.407 .918 & 254.884 .000 \\
\hline 1983 & 397.775 & 205.639 .000 & 1.528 .601 & 269.878 .000 \\
\hline 1984 & 478.492 & 223.976 .000 & 1.360 .697 & 346.364 .000 \\
\hline 1985 & 474.433 & 218.815 .000 & 1.446 .703 & 352.463 .000 \\
\hline 1986 & 626.817 & 227.158 .000 & 1.448 .150 & 382.295 .000 \\
\hline 1987 & 775.401 & 254.122 .000 & 1.770 .260 & 424.442 .000 \\
\hline 1988 & 1.116 .879 & 322.427 .000 & 1.891 .096 & 459.542 .000 \\
\hline 1989 & 1.430 .173 & 363.812 .000 & 1.832 .151 & 492.922 .000 \\
\hline 1990 & 1.589 .492 & 393.592 .000 & 2.071 .398 & 516.987 .000 \\
\hline 1991 & 1.769 .587 & 421.730 .000 & 2.152 .229 & 508.363 .000 \\
\hline 1992 & 2.050 .235 & 448.163 .000 & 2.139 .957 & 553.923 .000 \\
\hline 1993 & 2.005 .078 & 464.773 .000 & 2.082 .128 & 603.438 .000 \\
\hline 1994 & 2.314 .721 & 512.627 .000 & 1.904 .164 & 689.215 .000 \\
\hline 1995 & 2.661 .338 & 584.743 .000 & 1.394 .391 & 770.852 .000 \\
\hline 1996 & 2.445 .384 & 625.073 .000 & 1.462 .129 & 822.025 .000 \\
\hline 1997 & 2.514 .400 & 689.182 .000 & 1.739 .227 & 899.020 .000 \\
\hline 1998 & 2.340 .999 & 682.138 .000 & 1.974 .952 & 944.353 .000 \\
\hline 1999 & 2.698 .039 & 695.797 .000 & 2.271 .582 & 1.059 .440 .000 \\
\hline 2000 & 3.252 .355 & 781.918 .000 & 2.551 .112 & 1.259 .300 .000 \\
\hline 2001 & 2.696 .348 & 729.100 .000 & 2.840 .351 & 1.179 .180 .000 \\
\hline 2002 & 2.644 .363 & 693.103 .000 & 2.746 .483 & 1.200 .230 .000 \\
\hline 2003 & 3.196 .572 & 724.771 .000 & 2.741 .977 & 1.303 .050 .000 \\
\hline 2004 & 584.185 & 814.875 .000 & 3.791 .270 & 1.525 .680 .000 \\
\hline 2005 & 939.814 & 901.082 .000 & 3.666 .722 & 1.732 .706 .000 \\
\hline 2006 & 1.535 .860 & 1.025 .967 .000 & 3.236 .923 & 1.918 .077 .000 \\
\hline 2007 & 2.036 .287 & 1.148 .199 .000 & 3.302 .188 & 2.020 .403 .000 \\
\hline 2008 & 2.850 .154 & 1.287 .442 .000 & 3.078 .390 & 2.169 .487 .000 \\
\hline
\end{tabular}




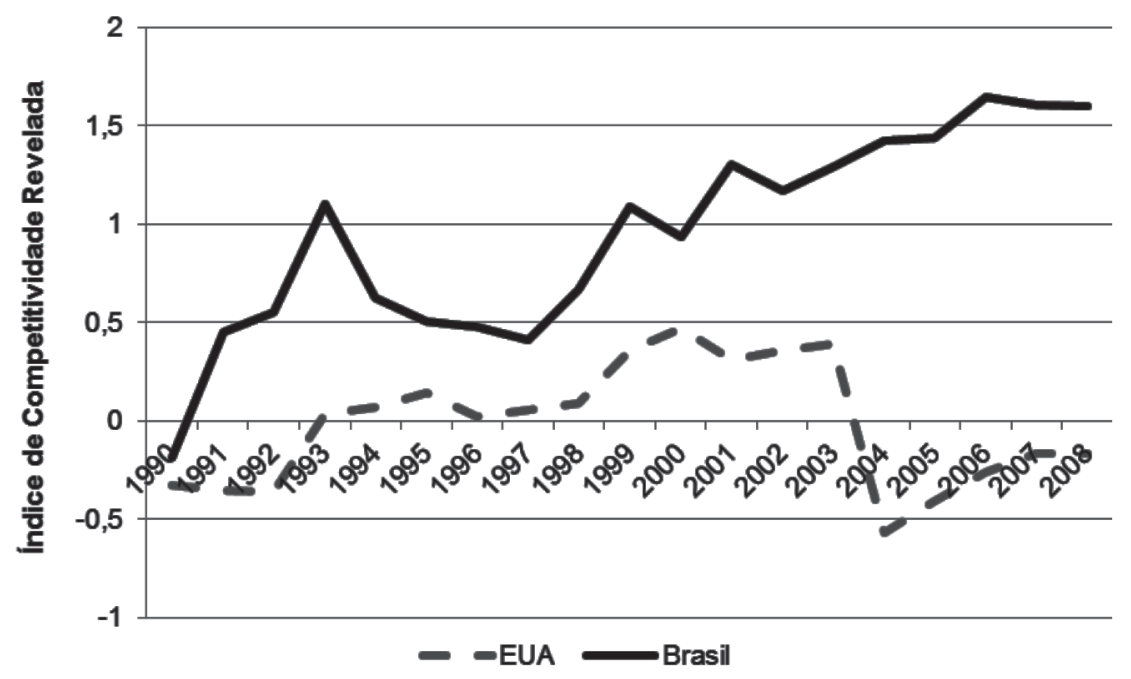

Figura 1. Índice de Competitividade Revelada da carne bovina do Brasil e dos EUA, elaborado com base nos dados da FAO (2011) e WTO (2011).

gradativamente, o CR, sendo o ano de 2006 o ápice da vantagem competitiva no mercado mundial $(\mathrm{CR}=1,643)$. Desde 2006, a Rússia aumentou as compras de carne bovina brasileira, tornando-se a principal compradora, e o Irã apresentou um importante crescimento nas importações desse produto, mantendo o Brasil como um dos principais exportadores (ABIEC, 2011).

Entre 2006 e 2008, o Índice manteve-se praticamente estável. Na ocorrência de uma reforma na política agrícola mundial, pode-se supor que o Brasil será favorecido. Por exemplo, um corte de $50 \%$ nas tarifas e subsídios à exportação mundial e a diminuição de 50\% no apoio doméstico à agricultura nos países da Organização para a Cooperação e Desenvolvimento Econômico (OCDE) proporcionariam um incremento de US $\$ 1,7$ bilhões nas receitas do país, cerca de $0,3 \%$ do PIB nacional (OECD/ WTO, 2011).

Cabe ressaltar que diversos outros fatores também podem ter influenciado a competitividade dos países, tais como os avanços tecnológicos, os insumos utilizados na produção, a qualidade do produto ofertado, os recursos naturais disponíveis, a taxa cambial, os impostos e os custos com transporte. Além disso, a metodologia utilizada nesse trabalho serve como um indicador para inferir sobre as possíveis causas do aumento ou diminuição da competitividade de países.

\section{CONCLUSÕES}

A comparação entre os índices de Competitividade Revelada demonstrou que o Brasil possuiu desempenho superior aos EUA em todos os períodos analisados. Devido a problemas de ordem sanitária e/ou econômica, ambos os países diminuíram seus índices em alguns momentos. Por outro lado, aumentaram suas vantagens quando acordos de cooperação foram efetivados. Mesmo que a produção de carne bovina dos EUA seja apoiada em subsídios governamentais, a carne bovina brasileira demonstrou ser mais competitiva.

\section{AGRADECIMENTOS}

Ao Centro de Estudos e Pesquisas em Agronegócios (CEPAN), ao Programa de Pós-Graduação em Agronegócios e ao Núcleo de Estudos em Sistemas de Produção de Bovinos de Corte e Cadeia Produtiva (NESPRO), pertencentes à Universidade Federal do Rio Grande do Sul UFRGS; e ao Conselho Nacional de Desenvolvimento Científico e Tecnológico (CNPq) pelo apoio financeiro para a realização desse trabalho.

\section{REFERÊNCIAS}

Andreatta T (2003) Febre aftosa no Rio Grande do Sul no ano de 2000: uma análise das transformações ocorridas nos sistemas de produção dos agricultores produtores de leite de Jóia. Dissertação de Mestrado. Programa de Pós-Graduação em Desenvolvimento Rural, Universidade Federal do Rio Grande do Sul, Porto Alegre, 266p.

ABIEC (2011) Associação Brasileira das Indústrias Exportadoras de Carne. Exportação brasileira de carne cresce com preço e volume maiores. Disponível em: 〈http://www.abiec.com.br/>. Acessado em: 27 de novembro de 2011.

Balassa B (1965) Trade liberalisation and revealed comparative advantage. Manchester School of Economic and Social Studies, 33:99-123.

Bismarck JVR (2004) OECD Scolds EU, U.S. for slow farm-subsidy cuts. The Wall Street Journal Europe. Disponível em: <http:// www.oecd.org/dataoecd/57/11/33920739.pdf>. Acessado em: 15 de julho de 2011.

Carvalho MA (1999) Comércio agropecuário brasileiro no MERCOSUL. Informações Econômicas, 29:7-22.

Carvalho MA (2001) Políticas públicas e competitividade da agricultura. Revista de Economia Política, 21:117-140. 
EWG (2011) Environmental Working Group. Farm subsidy database. Disponível em: <http://farm.ewg.org/ subsidyprimer.php>. Acessado em: 16 de agosto de 2011.

FAO (2011) Food and Agriculture Organization of the United Nations. TradeSTAT - detailed trade data. Disponível em: <http:/ /faostat.fao.org/>. Acessado em: 23 de novembro de 2011.

Henneberry SR \& Mutondo JE (2009) Agricultural trade among NAFTA countries: a case study of US meat exports. Applied Economic Perspectives and Policy, 31:424-445.

Jank MS, Nassar AM \& Tachinardi MH (2004) Agronegócio e comércio exterior brasileiro. Revista USP, 64:14-27.

Machado TA, Ilha AS \& Rubim LS (2007) Competitividade da carne bovina brasileira no mercado internacional (1994-2002). Cadernos PROLAM/USP, 1:87-101.

Martinelli LA, Naylor R, Vitousek PM \& Moutinho P (2010) Agriculture in Brazil: impacts, costs, and opportunities for a sustainable future. Current Opinion in Environmental Sustainability, 2:431-438.

Milner HV \& Kubota K (2004) Why the move to free trade? Democracy and trade policy in the developing countries. Disponível em: <http://www.princeton.edu/ hmilner/ forthcoming\%20papers/LDCdem_IO.pdf >. Acessado em: 04 de maio de 2011.

MAPA (2001) Ministério da Agricultura e Abastecimento. Emergência sanitária no Estado do Rio Grande do Sul: relatórios sobre as ações executadas para a eliminação dos focos de febre aftosa ocorridas no ano de 2000. Brasília, MAPA. 63p.

MAPA (2007) Ministério da Agricultura Pecuária e Abastecimento. Cadeia produtiva de carne bovina. Brasília, IICA, MAPA/ SPA. 86p.

OECD (2005) Organisation for Economic Co-Operation and Development. Agricultural policy reform in Brazil. Disponível em: <http://www.oecd.org/>. Acessado em: 04 de maio de 2011.

OECD (2010) Organisation for Economic Co-Operation and Development .Economic, environmental and social statistics. Disponível em: <http://www.oecd-ilibrary.org/statistics>. Acessado em: 19 de junho de 2011.

OECD/WTO (2011) Organisation for Economic Co-Operation and Development/ World Trade Organisation. Aid for trade at a glance 2011: Showing results. Paris, OECD Publishing. 400p.
Opp MM (2010) Tariff wars in the Ricardian Model with a continuum of goods. Journal of International Economics, $80: 212-225$.

Paula SRL \& Faveret Filho P (2001) Exportações de carne bovina: desempenho e perspectivas. Rio de Janeiro, BNDES Setorial, 14:27-46.

Polaquini LEM, Souza JG \& Gebara JJ (2006) Transformações técnico-produtivas e comerciais na pecuária de corte brasileira a partir da década de 90. Revista Brasileira de Zootecnia, 35:321327.

Reis JD (2008) Análise do crescimento das exportações brasileiras de carne bovina entre 1990 e 2002: uma aplicação do modelo constant market share. Revista Ceres, 55:179-186.

Ricardo D (1817) On the principles of political economy and taxation. London, John Murray, Albemarle-Street. 333p.

USDA (2010) United Stated Department of Agriculture. Livestock and poultry: world markets and trade. Disponível em: < http:// w w w. f a s.u s d a.gov/d 1 p/ c i r c u 1 a r / $2010 /$ livestock_poultryfull101510.pdf >. Acessado em: 14 de agosto de 2012 .

USDA (2012) United Stated Department of Agriculture. Livestock and poultry: world markets and trade. Disponível em: < http:// usda01.library.cornell.edu/usda/current/livestock-poultry-ma/ livestock-poultry-ma-10-18-2012.pdf >. Acessado em: 22 de agosto de 2012.

Waquil PD, Alvim AM, Silva LX \& Trapp GP (2004) Vantagens comparativas reveladas e orientação regional das exportações agrícolas brasileiras para a União Europeia. Revista de Economia e Agronegócio, 2:137-159.

WTO (2011) World Trade Organisation. Statistics database: time series on international trade. Disponível em: <http:// www.wto.org/index.htm>. Acessado em: 30 de setembro de 2012.

WWF (2010) World Wide Fund for Nature. Living planet report 2010: biodiversity, biocapacity and development. Disponível em: < http://www.ourplanet.com/livingplanetreport/2010/ 1_About\%20the\%20LPR.pdf >. Acessado em: 03 de março de 2012 . 